\title{
Giant Geometrically Amplified Piezoresistance in Metal-Semiconductor Hybrid
} Resistors

\author{
Hansen, Ole; Reck, Kasper; Thomsen, Erik Vilain
}

Published in:

Journal of Applied Physics

Link to article, DOI:

$10.1063 / 1.3033558$

Publication date:

2008

Document Version

Publisher's PDF, also known as Version of record

Link back to DTU Orbit

Citation (APA):

Hansen, O., Reck, K., \& Thomsen, E. V. (2008). Giant Geometrically Amplified Piezoresistance in MetalSemiconductor Hybrid Resistors. Journal of Applied Physics, 104(11), 114510.

https://doi.org/10.1063/1.3033558

\section{General rights}

Copyright and moral rights for the publications made accessible in the public portal are retained by the authors and/or other copyright owners and it is a condition of accessing publications that users recognise and abide by the legal requirements associated with these rights.

- Users may download and print one copy of any publication from the public portal for the purpose of private study or research.

- You may not further distribute the material or use it for any profit-making activity or commercial gain

- You may freely distribute the URL identifying the publication in the public portal

If you believe that this document breaches copyright please contact us providing details, and we will remove access to the work immediately and investigate your claim. 


\title{
Giant geometrically amplified piezoresistance in metal-semiconductor hybrid resistors
}

\author{
Ole Hansen, ${ }^{\text {a) }}$ Kasper Reck, and Erik V. Thomsen \\ Department of Micro- and Nanotechnology, DTU Nanotech, Technical University of Denmark, \\ Building 345E, DK-2800 Kgs. Lyngby, Denmark
}

(Received 15 August 2008; accepted 16 October 2008; published online 8 December 2008)

\begin{abstract}
We show that very high geometrically amplified piezoresistance can indeed be obtained in microstructured metal-semiconductor hybrid devices, even significantly higher amplification factors than the factor of approximately 8 demonstrated recently by Rowe and co-workers may be achieved. However, we also show that this amplification cannot be used to realize high sensitivity sensor devices due to limitation of the applied voltage across the device when the transfer resistance is smaller than the total resistance of the device. In that case, the sensitivity in units of $\mathrm{V} \mathrm{V}^{-1} \mathrm{~Pa}^{-1}$ is always less than the sensitivity of conventional piezoresistors fabricated in the same piezoresistive material. () 2008 American Institute of Physics. [DOI: 10.1063/1.3033558]
\end{abstract}

\section{INTRODUCTION}

In 1954, Smith ${ }^{1}$ reported the first measurements of the piezoresistance coefficients of silicon in his seminal paper. Since then simple silicon piezoresistors have been used for stress sensing in a wide variety of microelectromechanical systems, including accelerometers, pressure sensors, and chemical sensors ${ }^{2-5}$ due to the large gauge factor in silicon compared with other materials. Micrometer scale silicon piezoresistors usually have gauge factors of approximately 120 or less, depending primarily on temperature, resistor orientation, doping level, and stress direction. Since an increased gauge factor usually directly translates to an increased sensor sensitivity, gauge factors increased beyond the native silicon value are useful for the next generation of highly sensitive sensors. Several reports of giant piezoresistive effects in $p$-type silicon nanowires (SiNWs) have appeared recently; gauge factors more than an order of magnitude larger than seen in conventional microscale piezoresistors were reported. ${ }^{6-8}$ It was convincingly suggested by Rowe ${ }^{9}$ that the giant piezoresistive effect in SiNWs originates from a stressinduced modulation of the surface depletion region due to changes in surface state charge and neutral level; hence, the observed large gauge factors may be difficult to apply to stable piezoresistive sensors. Recently, Rowe et al. ${ }^{10}$ showed that giant gauge factors may be realized in microscale devices. By combining a conventional silicon piezoresistor with an aluminum shunt into a metal-silicon hybrid piezoresistor, a giant geometrical amplification of the piezoresistive effect may be obtained; a gauge factor of impressive 843 was shown. ${ }^{10}$ The piezoresistance boost in metal-semiconductor hybrid resistors is qualitatively identical to an observed boost, extraordinary magnetoresistance, in the magnetoresistance of the same type of structures. ${ }^{11,12}$ Contrary to SiNW

\footnotetext{
${ }^{a}$ Electronic mail: ole.hansen@nanotech.dtu.dk. Also at CINF Center for Individual Nanoparticle Functionality, Technical University of Denmark, Building 345E, DK-2800 Kgs. Lyngby, Denmark.
}

piezoresistors, the metal-silicon hybrid piezoresistor may easily be fabricated using conventional UV lithography and thus lends itself to mass production.

We present an analytical solution to the transfer resistance of a simplified metal-silicon hybrid piezoresistor and show that large geometrical amplification may be obtained. However, we also show that the geometrical amplification comes at the cost of an increased ratio between the applied voltage and the measured voltage. Finally, we show that the effective sensitivity of conventional silicon piezoresistors remains superior to that of metal-silicon hybrids. The analytical results are compared to finite element model (FEM) calculations.

\section{THEORY}

In practical stress measurements based on conventional piezoresistive sensors the useful sensitivity is maximized ${ }^{13}$ in a measurement setup as shown in Fig. 1(a), where a constant current $I_{0}$ is forced through a simple resistor (here shown as a transfer resistor for generality) of aspect ratio $L / W$, while the voltage drop $V$ across the resistor is monitored at the relative position $L_{V} / L$ and the change in voltage drop $\Delta V$ caused by the applied uniaxial stress $\sigma$ represents the real

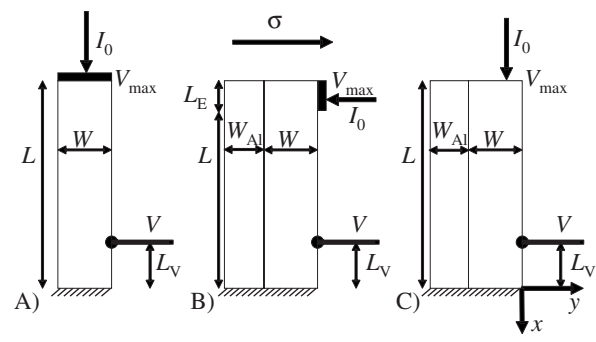

FIG. 1. Schematic top view of the different piezoresistor topologies considered. In (a) a simple transfer resistor with the aspect ratio $L / W$ and a voltage measurement point at $L_{V}$ is shown. (b) shows a schematic of half of the symmetrical metal-semiconductor hybrid transfer resistor used in Ref. 10, where one side of the resistor is in contact with a highly conducting metal bar of width $W_{\mathrm{Al}}$. (c) shows a simplified metal-semiconductor hybrid transfer resistor. A unidirectional stress $\sigma$ is applied normal to the long axis of the resistors. 
measurement signal. The magnitude of the incremental voltage drop is limited since the magnitude of the voltage drop across the full resistor structure is limited to some maximum value due to material limitations (e.g., dielectric breakdown), application limitations (e.g., Joule heating), and instrument limitations. It follows that a proper comparison of piezoresistive sensors should be based on evaluation of $\Delta V /\left(V_{\max } \Delta \sigma\right)=\left(V / V_{\max }\right) \Delta R /(R \Delta \sigma)$, where $V_{\max }$ is the maximum voltage across the sensor structure and $\Delta \sigma$ is the change in uniaxial stress to be measured, while $\Delta R$ is the stress induced change in transfer resistance $R=V / I_{0}$. For the structure shown in Fig. 1(a) $\Delta V /\left(V_{\max } \Delta \sigma\right)=\left(L_{V} / L\right) \Pi_{\text {eff }}$ as is easily verified; $\Pi_{\mathrm{eff}}=\Delta R /(R \Delta \sigma)$ is the effective piezoresistance coefficient for the particular material and arrangement of current and stress directions, while the geometrical factor $L_{V} / L=V / V_{\max }$. Obviously, a simple resistor with $L_{V}=L$ is the better arrangement.

\section{A. Metal-semiconductor hybrid transfer resistors}

In Fig. 1(b) half of the symmetrical metal-semiconductor hybrid transfer resistor used by Rowe et al. ${ }^{10}$ is shown schematically; for simplicity, symmetry has been used to introduce an isopotential ground plane. The bar shaped transfer resistor is in contact with a metal bar of width $W_{\mathrm{Al}}$ along one side, while a current supply contact of length $L_{E}$ is placed on the opposite side; this arrangement was experimentally shown to cause a geometrical amplification of the piezoresistive response $\Delta R /(R \Delta \sigma) .{ }^{10}$ In Fig. 1(c) a simplified transfer resistance structure without the contact is shown. This may easily be analyzed analytically if a current is forced through the top boundary in contrast to the structure in Fig. 1(b) where an analytical analysis is not equally straightforward.

We shall assume that the devices are realized in a homogeneous silicon film of thickness $h$ and with a stress free resistivity $\varrho_{0}$. The uniaxial stress $\sigma$ is assumed to be along the $y$-axis in a Cartesian coordinate system aligned to the resistor bar with the long axis along the $x$-axis. In this coordinate system the resistivity tensor is then diagonal with the nonzero elements $\varrho_{x x} \equiv \varrho_{t}=\varrho_{0}\left(1+\Pi_{t} \sigma\right), \quad \varrho_{y y} \equiv \varrho_{\ell}=\varrho_{0}(1$ $\left.+\Pi_{\ell} \sigma\right)$, and $\varrho_{z z} \equiv \varrho_{n}=\varrho_{0}\left(1+\Pi_{n} \sigma\right)$, where $\Pi_{t}, \Pi_{\ell}$, and $\Pi_{n}$ are the transversal, the longitudinal, and the normal piezoresistance coefficients, respectively. A purely two-dimensional current density $\mathbf{J}$ may thus be assumed for these structures. Since the current density is divergence free $\nabla \cdot \mathbf{J}=0$, the twodimensional electrostatic potential $\Phi(x, y)$ must satisfy

$$
\frac{1}{\varrho_{t}} \frac{\partial^{2} \Phi}{\partial x^{2}}+\frac{1}{\varrho_{\ell}} \frac{\partial^{2} \Phi}{\partial y^{2}}=0
$$

and proper boundary conditions. Obviously, proper scaling of the coordinates may turn Eq. (1) into a simple Laplacian, from which transfer resistances are given by $R$ $=\left(\sqrt{\varrho_{t} \varrho_{\ell}} / h\right) \mathcal{R}\left(\sqrt{\varrho_{t} / \varrho_{\ell}}\right)$ results; here $\mathcal{R}(u)$ is a normalized resistance function with geometry parameters such as the aspect ratios $L / W, L_{V} / W$, and $L_{E} / W$ and a geometry dependent topology. Thus the relative stress sensitivity of the transfer resistance in general becomes

$$
\frac{1}{R} \frac{\partial R}{\partial \sigma}=\frac{1}{2}\left(\Pi_{\ell}+\Pi_{t}\right)+\frac{1}{2}\left(\Pi_{\ell}-\Pi_{t}\right) S_{G},
$$

where $S_{G}$ is the geometrical amplification factor defined by

$$
S_{G} \equiv-\frac{1}{\mathcal{R}(u)} \frac{d \mathcal{R}(u)}{d u}
$$

with $u=\sqrt{\varrho_{t} / \varrho_{\ell}}$. The significance of $S_{G}$ may be illustrated by considering the case of $p$-type silicon with the stress along a $\langle 110\rangle$ direction and current flow in a $\{001\}$ plane, then the longitudinal and transversal piezoresistance coefficient magnitudes are approximately equal $\Pi_{\ell} \simeq-\Pi_{t}$ (since $\Pi_{\ell}=71.8$ $\times 10^{-11} \mathrm{~Pa}^{-1}$ and $\Pi_{t}=-66.3 \times 10^{-11} \mathrm{~Pa}^{-1}$ their magnitudes are within $4 \%$ of the mean magnitude ${ }^{1}$ ), and we may, for simplicity, take $\Pi_{\ell}=-\Pi_{t}=\Pi_{0}$; hence the relative stress sensitivity of the transfer resistance is proportional to the geometrical amplification factor and the piezocoefficient magnitude only $(\partial R / \partial \sigma) / R=\Pi_{0} S_{G}$. For simple transfer resistors $S_{G}= \pm 1$.

For the structure in Fig. 1(c) the boundary conditions are $\Phi(x,-W)=\Phi(0, y)=0, J_{y}(x, 0)=0$, and $J_{x}(-L, y)=J_{0}(y)$, if zero resistivity of the metal bar is assumed; $J_{0}(y)$ is the current density injected at the top boundary of the structure. In the interest of mathematical simplicity, we shall assume that the injected current density matches the first Fourier mode of the structure. The resulting potential is then

$$
\Phi(x, y)=\frac{-I_{0} \sqrt{\varrho_{t} \varrho_{\ell}}}{h} \frac{\sinh \left(\sqrt{\frac{\varrho_{t}}{\varrho_{\ell}}} \frac{\pi x}{2 W}\right)}{\cosh \left(\sqrt{\frac{\varrho_{t}}{\varrho_{\ell}}} \frac{\pi L}{2 W}\right)} \cos \left(\frac{\pi y}{2 W}\right) .
$$

It follows that the transfer resistance becomes

$$
R=\frac{\Phi\left(-L_{V}, 0\right)}{I_{0}}=\frac{\sqrt{\varrho_{t} \varrho_{\ell}}}{h} \frac{\sinh \left(\sqrt{\frac{\varrho_{t}}{\varrho_{\ell}}} \frac{\pi L_{V}}{2 W}\right)}{\cosh \left(\sqrt{\frac{\varrho_{t}}{\varrho_{\ell}}} \frac{\pi L}{2 W}\right)}
$$

in agreement with the general resistivity dependency of the transfer resistance. The geometrical amplification factor is easily obtained by differentiation

$$
S_{G}=\frac{\pi L}{2 W} \tanh \left(\frac{\pi L}{2 W}\right)-\frac{\pi L_{V}}{2 W} \operatorname{coth}\left(\frac{\pi L_{V}}{2 W}\right) .
$$

At sufficiently large values of the aspect ratios, $L / W$ and $L_{V} / W$, the geometrical amplification factor may be approximated by $S_{G} \simeq \pi\left(L-L_{V}\right) /(2 W)$, while at low values of the aspect ratios $S_{G} \simeq-1$, as expected. Obviously, very large geometrical amplification factors may be obtained by proper selection of the aspect ratios.

The potential ratio $V / V_{\max }=\Phi\left(-L_{V}, 0\right) / \Phi(-L, 0)$ of the voltage at the voltage measurement point to the maximum voltage across the structure obtained from Eq. (4) is 


$$
\frac{V}{V_{\max }}=\frac{\sinh \left(\sqrt{\frac{\varrho_{t}}{\varrho_{\ell}}} \frac{\pi L_{V}}{2 W}\right)}{\sinh \left(\sqrt{\frac{\varrho_{t}}{\varrho_{\ell}}} \frac{\pi L}{2 W}\right)} \simeq \exp \left[-\sqrt{\frac{\varrho_{t}}{\varrho_{\ell}}} \frac{\pi\left(L-L_{V}\right)}{2 W}\right]
$$

The approximation is valid at sufficiently large values of the aspect ratios $L / W$ and $L_{V} / W$, where extremely low values of the potential ratio may result. Unfortunately, the tall price paid in terms of low voltage ratio in order to obtain high geometrical amplification more than offsets the advantage gained since the proper dimensionless amplification factor is $S_{G} V / V_{\max }$ for which the simple model predicts $S_{G} V / V_{\max }$ $\leq 1 / e$. Note, a large $S_{G}$ is intimately linked to a strong position dependency of $V / V_{\max }$.

\section{B. Conformal mapping}

Support of conclusions based on the simple derivation above may be obtained by analyzing the resistor structures using conformal mapping techniques. ${ }^{14}$ The structure in Fig. 1(b) may be mapped on a simple transfer resistor as described by Trefethen; ${ }^{15}$ the mapping, however, is rather inconvenient for simple analysis due to the elliptic integrals involved. From such mapping the total current in the resistor may be approximated by

$$
I_{0} \simeq \frac{h V_{\max }}{\sqrt{\varrho_{t} \varrho_{\ell}}}\left(\frac{L_{E}}{W} \sqrt{\frac{\varrho_{t}}{\varrho_{\ell}}}+\alpha\right)
$$

with $\alpha \simeq 0.4$ accounting for the spreading current. An equally simple approximation to the potential at the voltage measurement point was not found. However, the potential in a structure similar to Fig. 1(b), but with an infinitely long extension $L_{E}$ fully covered by an isopotential current supply contact, may easily be determined using the conformal mapping

$$
z_{2}=\frac{2 V_{\max }}{\pi} \arcsin \frac{\sinh \left(\frac{\pi z}{2 W} \sqrt{\frac{\varrho_{t}}{\varrho_{\ell}}}\right)}{\sinh \left(\frac{\pi L}{2 W} \sqrt{\frac{\varrho_{t}}{\varrho_{\ell}}}\right)}
$$

Here $z=x+i y \sqrt{\varrho_{\ell} / \varrho_{\mathrm{t}}}$ represents the position in the resistor structure, while $z_{2}=\Phi+i \Psi$ is the potential-flux function, with $\Phi$ as the potential and $\Psi$ as the flux function, while $i=\sqrt{-1}$. The resulting voltage ratio may be used as a good approximation to the voltage ratio even in the structure with finite contact length

$$
\begin{aligned}
\frac{V}{V_{\max }} & =\frac{2}{\pi} \arcsin \frac{\sinh \left(\frac{\pi L_{V}}{2 W} \sqrt{\frac{\varrho_{t}}{\varrho_{\ell}}}\right)}{\sinh \left(\frac{\pi L}{2 W} \sqrt{\frac{\varrho_{t}}{\varrho_{\ell}}}\right)} \\
& \simeq \frac{2}{\pi} \frac{\sinh \left(\frac{\pi L_{V}}{2 W} \sqrt{\frac{\varrho_{t}}{\varrho_{\ell}}}\right)}{\sinh \left(\frac{\pi L}{2 W} \sqrt{\frac{\varrho_{t}}{\varrho_{\ell}}}\right)},
\end{aligned}
$$

where the approximation is valid if $L_{V} \ll L$, and differ from Eq. (7) only by the prefactor $2 / \pi$. Calculations of $S_{G}$ based on Eqs. (8) and (10) agree well with Eq. (6).

\section{Finite metal resistivity}

A first order correction for finite resistivity $\varrho_{\mathrm{Al}}$ of the metal bar is obtained by noting that the most of the current is flowing in the metal (thickness $h_{\mathrm{Al}}$ ). Thus a correction potential $\delta \Phi(x)=-I_{0} \varrho_{\mathrm{Al}} x /\left(h_{\mathrm{Al}} W_{\mathrm{Al}}\right)$ may be added to the potential equation (4) and the potential leading to Eq. (10). If the metal resistivity is assumed unaffected by stress the effective geometrical amplification factor $S_{G \text { eff }}$ corrected for finite metal resistivity becomes $S_{G \text { eff }}=S_{G} /\left(1+R_{\mathrm{Al}} / R\right)$, with $R_{\mathrm{Al}}$ $=\varrho_{\mathrm{Al}} L_{V} /\left(h_{\mathrm{Al}} W_{\mathrm{Al}}\right)$. This severely limits the effective geometrical amplification at high aspect ratios where extremely low values of the transfer resistance results.

\section{FEM AND DISCUSSION}

A FEM of the metal-semiconductor hybrid resistor structure was implemented in COMSOL MULTIPHYSICS 3.3 using a stress free silicon resistivity of $\varrho_{0}=0.1 \Omega \mathrm{cm}$ and using Smith's piezocoefficients ${ }^{1}$ to model the stress dependence, while the resistivity of $\mathrm{Al}$ was assumed to be $\varrho_{\mathrm{Al}}=2.65$ $\times 10^{-6} \Omega \mathrm{cm}$. A configuration identical to the sketch in Fig. 1(b) was implemented with the aluminum width equal to the active resistor length $W_{\mathrm{Al}}=L$, and a thickness equal to the silicon thickness $h_{\mathrm{Al}}=h$, while the length of the current supply contact was $L_{E}=L / 10$. The position of the voltage measurement point was varied such that $L_{V} / L$ $\in[1 / 10,1 / 3,1 / 2,2 / 3]$, and the relative resistor width varied in a wide range $W / L$. These calculations were repeated with $\mathrm{Al}$ replaced by a perfect conductor.

Figure 2 shows the voltage ratio $V / V_{\max }$ from the FEM calculations as a function of $\left(L-L_{V}\right) / W$ (full symbols: finite metal resistivity and open symbols: zero metal resistivity) along with curves calculated from Eq. (10) corrected for finite $\mathrm{Al}$ resistivity (full lines). Except at low values of $\left(L-L_{V}\right) / W$ all data and curves can hardly be distinguished, only data for $L_{V} / L=1 / 10$ separate slightly from the rest. In all cases, Eq. (10) approximates the FEM data very well. The effect of finite $\mathrm{Al}$ resistivity is visible at large $L / W$ ratios. The simple result [Eq. (7)] (dashed line) is shown for comparison and is seen to be in fair agreement with the FEM data.

Figure 3 shows the geometrical amplification factor $S_{G}$ from the FEM calculations as a function of $\left(L-L_{V}\right) / W$ (full symbols: finite metal resistivity and open symbols: zero 


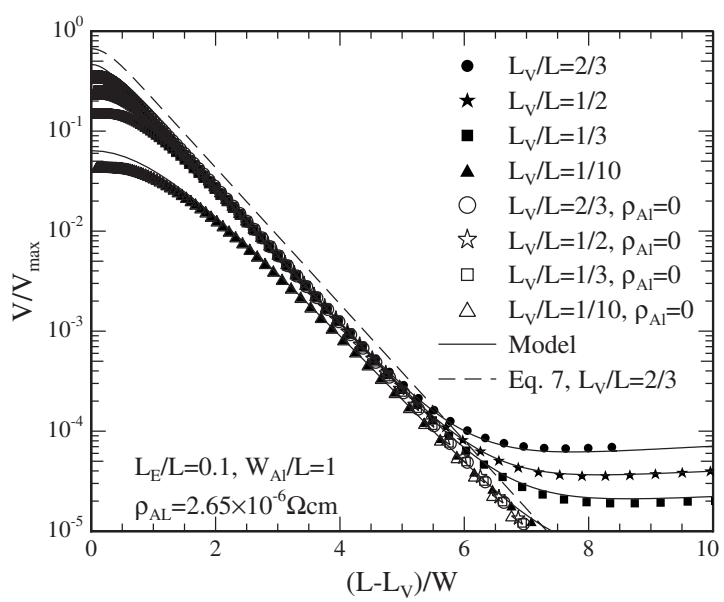

FIG. 2. Voltage ratio $V / V_{\max }$ as a function of $\left(L-L_{V}\right) / W$ calculated using the COMSOL FEM model. Data from calculations using $L_{E} / L=0.1, W_{\mathrm{Al}} / L$ $=1$, and $L_{V} / L \in[1 / 10,1 / 3,1 / 2,2 / 3]$ (symbols $[\boldsymbol{\Delta , \boldsymbol { \square } , \star , \bullet}]$ ) are shown along with the model [Eq. (10)] (full lines) including corrections for finite $\mathrm{Al}$ resistivity. The corresponding open symbols signify FEM data assuming zero metal resistivity. Equation (7) (dashed line) at $L_{V} / L=2 / 3$ is shown for comparison.

metal resistivity) along with model calculations using Eqs. (10) and (8) corrected for finite Al resistivity (full lines) and the simple result [Eq. (6)] (dashed lines). All perfect conductor FEM data group in a narrow band with $S_{G}$ increasing somewhat faster with increasing $\left(L-L_{V}\right) / W$ than the simple model predicts; the reason is a somewhat stronger position dependency of the potential in the real structure compared to that of the simplified model structure as verified by FEM data. Finite Al resistivity limits the magnitude of $S_{G}$ to some peak value at moderate $\left(L-L_{V}\right) / W$ in agreement with the model; the actual value depends on the resistivity ratio $\varrho_{0} / \varrho_{\mathrm{Al}}$ and increases when it is increased. Obviously, quite large geometrical amplification is possible.

Finally, Fig. 4 shows the proper quality measure $S_{G} V / V_{\max }$ as a function of $\left(L-L_{V}\right) / W$. The general trend of FEM (full symbols: finite metal resistivity and open sym-

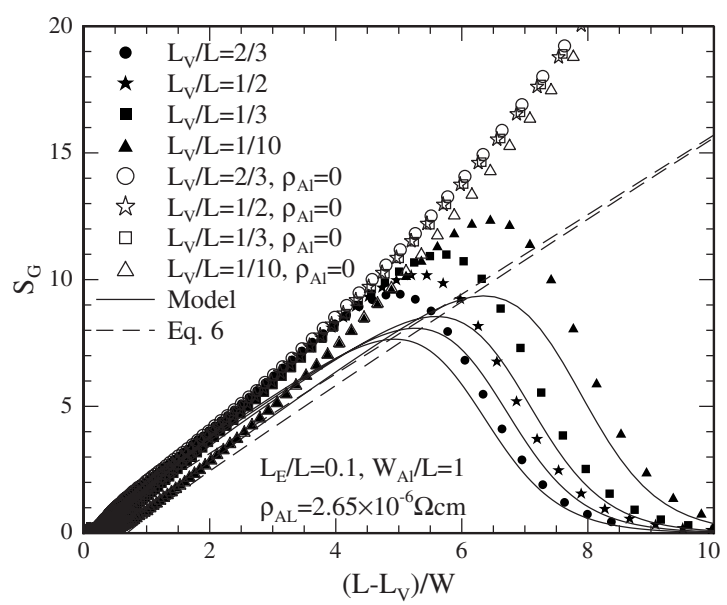

FIG. 3. Geometrical amplification $S_{G}$ as a function of $\left(L-L_{V}\right) / W$ calculated using the COMSOL FEM model. Data from calculations using $L_{E} / L=0.1$ and $L_{V} / L \in[1 / 10,1 / 2,1 / 2,2 / 3]$ (symbols $[\boldsymbol{\Delta}, \boldsymbol{\square}, \star, \bullet]$ ) are shown along with the simple model [Eq. (6)] (dashed lines), as well as a model using Eqs. (8) and (10) corrected for finite Al resistivity (full lines). The corresponding open symbols signify FEM data assuming zero metal resistivity.

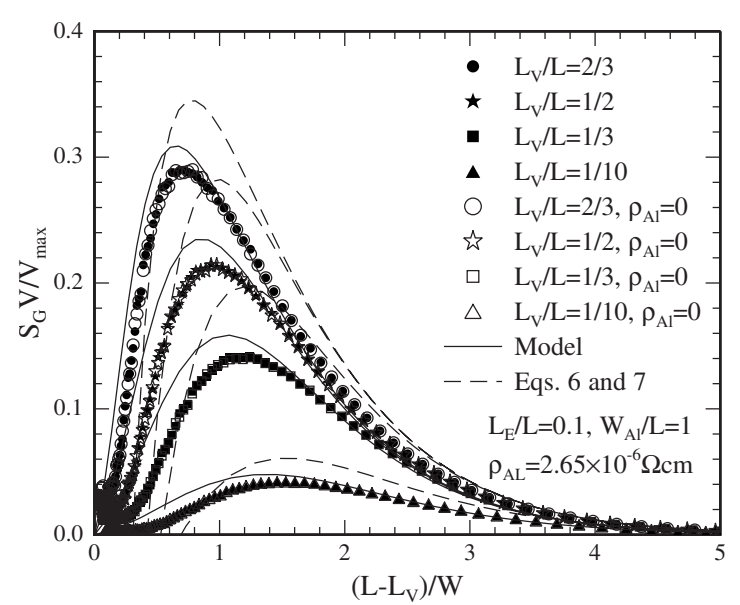

FIG. 4. Product of voltage ratio and geometrical amplification $S_{G} V / V_{\max }$ as a function of $\left(L-L_{V}\right) / W$ calculated using the COMSOL FEM model. Data from calculations using $L_{E} / L=0.1$ and $L_{V} / L \in[1 / 10,1 / 2,1 / 2,2 / 3]$ (symbols $[\mathbf{\Lambda}, \boldsymbol{\square}, \star, \bullet])$ are shown along with the simple model [Eqs. (6) and (7)] (dashed lines), as well as a model using Eqs. (8) and (10) corrected for finite $\mathrm{Al}$ conductivity (full lines). The corresponding open symbols signify FEM data assuming zero metal resistivity.

bols: zero metal resistivity) and model calculations (full and dashed lines) agree, and the effect of finite $\mathrm{Al}$ resistivity is hardly visible. The important point here is that for the metalsemiconductor hybrid, the magnitude of $S_{G} V / V_{\max }$ is smaller than both that of a simple piezoresistor $\left(S_{G} V / V_{\max }= \pm 1\right)$ and that of a simple transfer piezoresistor $\left(S_{G} V / V_{\max }= \pm L_{V} / L\right)$. FEM model calculations on devices with a finite size of the voltage measurement contact agree with the data shown.

\section{CONCLUSION}

We have shown analytically that a giant geometrically amplified piezoresistive response $\Delta R /(R \Delta \sigma)$ is indeed possible in metal-semiconductor hybrid resistor structures, but the advantage gained is more than lost due to the very low ratio of the measured voltage to the full applied voltage or, equivalently, due to the extremely low transfer resistance of the structure. Moreover, we note that the noise resistance of the hybrid structure may be significantly larger than the transfer resistance, thus the resolution will suffer. It follows that even though the geometrical amplification effect is interesting, hybrid structures in which the transfer resistance is smaller than the total resistance will be of little practical use since conventional piezoresistors have superior sensitivity and resolution.

\section{ACKNOWLEDGMENTS}

Center for Individual Nanoparticle Functionality (CINF) is sponsored by The Danish National Research Foundation.

${ }^{1}$ C. S. Smith, Phys. Rev. 94, 42 (1954).

${ }^{2}$ S. Huang, X. Li, Z. Song, Y. Wang, H. Yang, L. Che, and J. Jiao, J. Micromech. Microeng. 15, 993 (2005).

${ }^{3}$ A. Berns, U. Buder, E. Obermeier, A. Wolter, and A. Leder, Sens. Actuators, A 132, 104 (2006).

${ }^{4}$ J. Bartholomeyczik, S. Brugger, P. Ruther, and O. Paul, IEEE Sens. J. 5, 872 (2005).

${ }^{5}$ H. Jensenius, J. Thaysen, A. A. Rasmussen, L. H. Veje, O. Hansen, and A. 
Boisen, Appl. Phys. Lett. 76, 2615 (2000).

${ }^{6}$ T. Toriyama, D. Funai, and S. Sugiyama, J. Appl. Phys. 93, 561 (2003).

${ }^{7}$ R. He and P. Yang, Nat. Nanotechnol. 1, 42 (2006).

${ }^{8}$ K. Reck, J. Richter, and O. Hansen, Proceedings of Nanotech Boston, June 2008 (CRC Press, Boca Raton, 2008), Vol. 1, p. 920.

${ }^{9}$ A. C. H. Rowe, Nat. Nanotechnol. 3, 311 (2008).

${ }^{10}$ A. C. H. Rowe, A. Donoso-Barrera, Ch. Renner, and S. Arscott, Phys Rev. Lett. 100, 145501 (2008).
${ }^{11}$ S. A. Solin, T. Thio, D. R. Hines, and J. Heremans, Science 289, 1530 (2000).

${ }^{12}$ T. Zhou, D. R. Hines, and S. A. Solin, Appl. Phys. Lett. 78, 667 (2001).

${ }^{13}$ S. Gift and B. Maundy, IEEE Trans. Circuits Syst., II: Express Briefs 53, 178 (2006)

${ }^{14}$ W. Kaplan, Introduction to Analytic Functions (Addison-Wesley, Reading, 1966).

${ }^{15}$ L. N. Trefethen, Z. Angew. Math. Phys. 35, 692 (1984). 